Editorial

\title{
High Precision X-Ray Measurements
}

\author{
Alessandro Scordo \\ Istituto Nazionale di Fisica Nucleare-Laboratori Nazionali di Frascati (LNF-INFN), Frascati, 00044 Roma, Italy; \\ alessandro.scordo@lnf.infn.it \\ Received: 17 June 2019; Accepted: 22 June 2019; Published: 25 June 2019 \\ check for \\ updates
}

Keywords: X-ray; XAS; XRF; multidisciplinarity; X-ray source facilities; material investigation; graphite crystals

Since their discovery in 1895, the detection of X-rays has had a strong impact and various applications in several fields of science and human life. Kiloelectronvolt (keV) photons are indeed the leading actors in a large variety of physics fields, and impressive efforts have been and are being done to develop new type of detectors and new techniques, aiming to obtain higher precision measurements of their energy, position and polarization.

Historically, new detectors and monochromators for synchrotron radiation high precision measurements were first made in Europe at the Frascati laboratories by a Italian-French team in the sixties, using the $1 \mathrm{GeV}$ electron synchrotron [1]; a decade later, these efforts were followed by the Italian team of Balzarotti and Bianconi [2].

Applications of $\mathrm{X}$-ray measurements are relevant in fundamental and nuclear physics, biophysics, medical research, molecular and surface structure of materials studies.

The aim of this special issue is to have a global overview, from these communities and research fields, of the most recent developments in X-ray detection techniques and their impact.

To accomplish this aim, the published papers provide high quality research results, giving an insight into the hot topics and the main applications of X-ray photons.

In the paper of Andreeva et al. [3], a detailed description on the possible use of Mössbauer spectroscopy in material surface investigations is given, with particular emphasis on the importance of the polarization analysis in reflectivity experiments; the authors show the advantages of the polarization selection to deliver high quality data, providing a better interpretation of the magnetic ordering in multilayer films.

The properties of materials, and in particular their crystalline phase, is investigated in the paper of Macis et al. [4], where the structural changes of $\mathrm{MoO}_{3}$ thin films upon annealing at different temperatures are investigated using X-ray Absorption Spectroscopy (XAS). The presented results are very promising and suggest the possibility of using such material as a hard, protective, transparent and conductive material in different technologies. It represents the first important advancement for many applications, in particular for the development of compact radio frequency (RF) accelerating devices made of copper.

Concerning biological samples analysis, the first high precision Ca K-edge X-ray Absorption Near Edge Spectroscopy (XANES) measurement of CaATP molecules was performed using the Slac storage ring of Stanford University [5], followed by the high precision X-ray spectroscopy measurements on MnATP molecules at the first $1.5 \mathrm{GeV}$ European synchrotron radiation facility using a large storage ring (ADONE, INFN, Laboratories of Frascati) [6].

The usage of XAS technique on biological samples is also the main subject of the paper from E. De Santis et al. [7], with a particular effort toward the possibility of performing XAS measurements on very diluted samples, with an absorber concentration at the micromolar level. The reported measurements, focused on the $\mathrm{Cu}$ (II)-ProIAPP (islet amyloid polypeptide) complexes 
under near-physiological and equimolar concentrations of $\mathrm{Cu}(\mathrm{II})$ and peptide, may have a strong medical impact related to the cell death in type 2 diabetes mellitus, with important consequences on human health.

Another possible medical application of $\mathrm{keV}$ photons is described in the paper of Makek et al. [8], where the performances of a single-layer scintillator pixel detector are reported; the obtained results demonstrate the possibility of detecting Compton gammas with an energy and timing resolution comparable to those achieved in photo-electric absorption measurements. In addition, the authors show how the detection and full reconstruction of such gammas is very interesting and promising in medical imaging, with particular emphasis on the proton emission tomography (PET) technique, where measurement of polarization correlations of annihilation photons may improve the required sensitivity leading to a reduction of the needed number of electronic channels and of the overall costs.

X-ray measurements are a perfect tool for testing fundamental principle of physics, like the Pauli exclusion principle (PEP); this is reported and described in the paper by K. Piscicchia et al. [9], where the results of the measurements performed by the Violation of the Pauli exclusion principle (VIP) experiment at the INFN underground Laboratories of Gran Sasso are presented. The photons coming from the atomic transitions of a copper sample are detected and analysed, looking for the possible existence of PEP violating electrons producing an anomalous X-ray signal corresponding to the transition from the $2 p$ level to the 1 s level when this last one is already occupied by two electrons. The authors provide the most recent limit on the PEP violation probability, together with a detailed explanation of the performed analysis.

X-rays coming from atomic transitions can be also used, in nuclear physics, to investigate the low energy interaction involving strange quarks by measurements of kaonic atoms; this possibility is explored and reported in the paper from Curceanu et al. [10], which provides an overview of the measurements of kaonic atoms performed at the Double Anular $\Phi$-factory for Nice Experiments (DA $\Phi N E)$ collider at LNF-INFN and on the X-ray detectors used in the experiments, like charged couple devices (CCDs) and silicon drift detectors (SDDs).

These latter devices are, nowadays, the best performing large area spectroscopic detectors in terms of energy resolutions, and their faster timing capability with respect to CCDs make them suitable for measurements in high background environments. The characterization of a set of SDDs, produced by Fondazione Bruno Kessler (FBK, Trento, Italy) and used in the SIDDHARTA-2 (Silicon Drift Detector for Hadronic Atom Research by Timing Application) experiment at the DA $\Phi$ NE collider, in terms of stability and linearity is given by Miliucci et al. [11]; in this work, a linear response within $1 \mathrm{eV}$ is reported for photons in the energy range $4-12 \mathrm{keV}$, for typical energy resolutions of $120 \mathrm{eV} @ 6 \mathrm{keV}$ Full Width at Half Maximum (FWHM).

A factor 50 improvement in the energy resolution, with respect to SDDs, can be achieved with the Bragg spectroscopy technique, with the drawback of waiving the large acceptances and efficiencies typical of solid state devices. The possibility of pushing this technique toward millimetric and isotropic sources, instead of the standard micrometric collimated ones, is explored by the VOXES (high resolution VOn hamos X-ray spectrometer using HAPG for Extended Sources) project and reported in the paper by Scordo et al. [12], where a new Bragg spectrometer with graphite mosaic crystals in the Von Hamos configuration is proposed. In this paper, results of the measurements of the copper and iron $\mathrm{K} \alpha_{1,2}$ performed with different mosaicity crystals are discussed, investigating the effect of both the mosaicity and the crystal thickness on the energy resolution when keeping the source dimension at the millimetre level. The obtained results are very promising and trigger the possibility of using such spectrometers for nuclear physics experiments, as well as material investigations and trace metals identification, with several important applications in other fields.

The above-mentioned research is based on the usage, as diffraction crystals, of mosaic highly annealed pyrolitic graphite (HAPG) and highly oriented pyrolitic graphite (HOPG) crystals. These objects are experiencing a very rapid expansion on the market, due to their extremely interesting mechanical properties and physical properties, like a small lattice constant, low thermal 
expansion coefficient and the possibility of depositing them on a substrate of any geometrical shape. These characteristics, together with the HAPG and HOPG production mechanisms, are presented by one of the main graphite crystal producers, the OPTIGRAPH GmbH company, in the paper by Grigorieva et al. [13].

All the above-mentioned papers clearly suggest how the possibility of having access to X-ray sources is mandatory to perform high quality research in various fields.

One possibility is given by the DAФNE-Light beam facility at INFN Laboratories of Frascati, described in detail by A. Balerna in [14]; in particular, the soft X-ray DXR1 beamline is presented, together with a description of the typical XAS applications that can be performed on site.

The DXR1 beamline started delivering beamtime to users at the end of 2004; more recently, a proposal for building a free electron laser (EuPRAXIA@SPARC_LAB) at the INFN Laboratories of Frascati is under consideration. The technical details and the beam characteristics achievable in the main experimental lines are the main subject of the paper by A. Balerna et al. [15].

All the papers included in this Special Issue contribute to underlining the importance of X-ray detection for a very broad range of physics topics; most of these topics are covered by the published works and several others are mentioned in the paper references, providing an interesting and very useful overview from these different communities and research fields, of the most recent developments in X-ray detection and their impact on fundamental research and societal applications.

The readers of this special issue may also find other review papers published in other special issues of Condensed Matter, focused in particular on synchrotron radiation techniques $[16,17]$ very interesting.

Acknowledgments: I express my thanks to the contributing authors of this Special Issue, and to the journal Condensed Matter and MDPI for their support during this work.

Conflicts of Interest: The author declares no conflict of interest.

\section{References}

1. Jaeglé, P.; Missoni, G.; Dhez, P. Study of the Absorption of Ultrasoft X Rays by Bismuth and Lead Using the Orbit Radiation of the Frascati Synchrotron. Phys. Rev. Lett. 1967, 18, 887. [CrossRef]

2. Balzarotti, A.; Bianconi, A.; Burattini, E.; Grandolfo, M.; Habel, R.; Piacentini, M. Core transitions from the Al $2 \mathrm{p}$ level in amorphous and crystalline $\mathrm{Al}_{2} \mathrm{O}_{3}$. Phys. Status Solidi B 1974, 63, 77-87. [CrossRef]

3. Andreeva, M.; Baulin, R.; Chumakov, A.; Kiseleva, T.; Rüffer, R. Polarization Analysis in Mössbauer Reflectometry with Synchrotron Mössbauer Source. Condens. Matter 2019, 4, 8. [CrossRef]

4. Macis, S.; Rezvani, J.; Davoli, I.; Cibin, G.; Spataro, B.; Scifo, J.; Faillace, L.; Marcelli, A. Structural Evolution of $\mathrm{MoO}_{3}$ Thin Films Deposited on Copper Substrates upon Annealing: An X-ray Absorption Spectroscopy Study. Condens. Matter 2019, 4, 41. [CrossRef]

5. Bianconi, A.; Doniach, S.; Lublin, D. X-ray Ca K edge of calcium adenosine triphosphate system and of simple Ca compunds. Chem. Phys. Lett. 1978, 59, 121-124. [CrossRef]

6. Belli, M.; Scafati, A.; Bianconi, A.; Mobilio, S.; Palladino, L.; Reale, A.; Burattini, E. X-ray absorption near edge structures (XANES) in simple and complex Mn compounds. Solid State Commun. 1980, 35, 355-361. [CrossRef]

7. De Santis, E.; Shardlow, E.; Stellato, F.; Proux, O.; Rossi, G.; Exley, C.; Morante, S. X-Ray Absorption Spectroscopy Measurements of Cu-ProIAPP Complexes at Physiological Concentrations. Condens. Matter 2019, 4, 13. [CrossRef]

8. Makek, M.; Bosnar, D.; Pavelić, L. Scintillator Pixel Detectors for Measurement of Compton Scattering. Condens. Matter 2019, 4, 24. [CrossRef]

9. Piscicchia, K.; Amirkhani, A.; Bartalucci, S.; Bertolucci, S.; Bazzi, M.; Bragadireanu, M.; Cargnelli, M.; Clozza, A.; Curceanu, C.; Del Grande, R.; et al. High Precision Test of the Pauli Exclusion Principle for Electrons. Condens. Matter 2019, 4, 45. [CrossRef]

10. Curceanu, C.; Amirkhani, A.; Baniahmad, A.; Bazzi, M.; Bellotti, G.; Berucci, C.; Bosnar, D.; Bragadireanu, M.; Cargnelli, M.; Clozza, A.; et al. X-ray Detectors for Kaonic Atoms Research at DAФNE. Condens. Matter 2019, 4, 42. [CrossRef] 
11. Miliucci, M.; Iliescu, M.; Amirkhani, A.; Bazzi, M.; Curceanu, C.; Fiorini, C.; Scordo, A.; Sirghi, F.; Zmeskal, J. Energy Response of Silicon Drift Detectors for Kaonic Atom Precision Measurements. Condens. Matter 2019, 4, 31. [CrossRef]

12. Scordo, A.; Curceanu, C.; Miliucci, M.; Sirghi, F.; Zmeskal, J. Pyrolitic Graphite Mosaic Crystal Thickness and Mosaicity Optimization for an Extended Source Von Hamos X-ray Spectrometer. Condens. Matter 2019, 4, 38. [CrossRef]

13. Grigorieva, I.; Antonov, A.; Gudi, G. Graphite Optics-Current Opportunities, Properties and Limits. Condens. Matter 2019, 4, 18. [CrossRef]

14. Balerna, A. DAFNE-Light DXR1 Soft X-Ray Synchrotron Radiation Beamline: Characteristics and XAFS Applications. Condens. Matter 2019, 4, 7. [CrossRef]

15. Balerna, A.; Bartocci, S.; Batignani, G.; Cianchi, A.; Chiadroni, E.; Coreno, M.; Cricenti, A.; Dabagov, S.; Di Cicco, A.; Faiferri, M.; et al. The Potential of EuPRAXIA@SPARC_LAB for Radiation Based Techniques. Condens. Matter 2019, 4, 30. [CrossRef]

16. Campi, G.; Bianconi, A. Evolution of Complexity in Out-of-Equilibrium Systems by Time-Resolved or Space-Resolved Synchrotron Radiation Techniques. Condens. Matter 2018, 4, 32. [CrossRef]

17. Baccolo, G.; Cibin, G.; Delmonte, B.; Hampai, D.; Marcelli, A.; Di Stefano, E.; Macis, S.; Maggi, V. The Contribution of Synchrotron Light for the Characterization of Atmospheric Mineral Dust in Deep Ice Cores: Preliminary Results from the Talos Dome Ice Core (East Antarctica). Condens. Matter 2018, 3, 25. [CrossRef]

(C) 2019 by the author. Licensee MDPI, Basel, Switzerland. This article is an open access article distributed under the terms and conditions of the Creative Commons Attribution (CC BY) license (http://creativecommons.org/licenses/by/4.0/). 